\title{
Optimierte Patientenauswahl für Ventile
}

Fragestellung: Welche diagnostische Wertigkeit hat eine Kombination aus computerbasierter Fissurenanalyse im hochauflösenden Computertomografie (HRCT) und bronchoskopischer Messung der Kollateralventilation zur Vorhersage einer Volumenreduktion nach Therapie mit Endobronchialventilen (EBV)?

Hintergrund: Das Fehlen von Kollateralventilation zwischen Zielund Nachbarlappen ist eine entscheidende Voraussetzung für den Erfolg einer EBV-Therapie. In der klinischen Routine stehen 2 diagnostische Optionen zur Verfügung; die Vollständigkeit der interlobären Fissuren im HRCT gilt als Surrogatparameter für die Abwesenheit von Kollateralventilation. Mit einem bronchoskopisch geführten Ballonkatheter („Chartis-Messung“) kann die Kollateralventilation direkt gemessen werden. Der optimale Einsatz dieser 2 Methoden im Evaluationsprozess ist noch Originalie

Koster TD, van Rikxoort EM, Huebner RH et al. Predicting Lung Volume Reduction after Endobronchial Valve Therapy is Maximized Using a Combination of Diagnostic Tools. Respiration. 2016;92(3):150-7.

Patienten und Methoden: CT-Daten von vier prospektiven Studien (217 Patienten) wurden gepoolt und mit semiautomatischer Software wurde in einem prozentualen Score („fissure completeness score“/ FCS) erfasst und mit einer Volumenreduktion $>350 \mathrm{ml}$ im Ziellappen als Goldstandard verglichen. In einer ROC-Analyse wurden optimale Grenzwerte für vollständige Fissuren (Responder) und unvollständige Fissuren (Non-Responder) ermittelt. Eine Subgruppe mit teilweise vollständigen Fissuren wurde identifiziert, in der die Fissurenanalyse eine geringe Treffsicherheit zeigte. In dieser Gruppe wurde der komplementäre Nutzen der Chartis-Messung bestimmt.

Ergebnisse: Als optimaler Grenzwert für eine vollständige Fissur ergab sich ein FCS $>95 \%$, für eine unvollständige Fissur ein FCS $<80 \%$. Eine teilweise vollständige Fissur ist damit durch einen FCS zwischen $80 \%$ und 95\% gekennzeichnet. Der positive Vorhersagewert (PPV) für vollständige Fissuren lag bei 88,1\%, der negative Vorhersagewert (NPV) bei 92,9\%, die Treffsicherheit bei 89,2\%. In der Gruppe mit teilweise vollständigen Fissuren zeigte die Chartis-Messung ein PPV von $82,3 \%$, ein NPV von $84,6 \%$ und eine Treffsicherheit von $83,3 \%$. analysiert. Die Vollständigeit der interlobären Fissuren
Schlussfolgerung: Die Kombination von Fissurenanalyse und Chartis-Messung bei unklaren Fissurenbefunden erlaubt eine optimale Patientenauswahl für eine Therapie mit EBV und reduziert gleichzeitig die Zahl der erforderlichen Bronchoskopien und damit die Belastung der Patienten.

\section{- Kommentar von Dr. med. Wolfgang Gesierich}

\section{Erfahrung und Spezialsoftware erforderlich}

Aus den Ergebnissen dieser Arbeit ergibt sich ein Algorithmus für die Beurteilung der Kollateralventilation: Vorrang erhält die Fissurenanalyse. Patienten mit vollständigen Fissuren im Zielllappen (FCS > 95\%) können direkt einer Ventilimplantation zugeführt werden, jene mit unvollständigen Fissuren (FCS < 80\%) können von einer Ventiltherapie ausgeschlossen werden. Nur in der Gruppe mit unvollständigen Fissuren (FCS 80-95\%) ist die Chartis-Messung nötig. Anzumerken ist die retrospektive Natur der Studie: Optimalerweise würde man sich eine prospektive Evaluation der Testcharakteristika eines neuen diagnostischen Verfahrens wünschen. Aufgrund der vorliegenden Evidenz $[1,2]$ scheint es aber ethisch nicht mehr vertretbar, bei belegter Kollateralventilation Ventile zu implantieren. Eine prospektive Validierung wird es also wahrscheinlich nicht geben. Der neue Algorithmus sollte daher rasch Eingang in die klinische Routine finden und auch in zukünftigen Studien zu den EBV in der Patientenauswahl herangezogen werden. Die Fallstricke der beiden Methoden sind dabei zu bedenken. Eine visuelle Fissurenanalyse erfordert Erfahrung und ist stark untersucherabhängig [3]. In dieser Studie wurde sie durch eine computerbasierte Analyse unterstützt. Wo Erfahrung und entsprechende Software nicht vorliegen, sollte die Delegation der Analyse an ein Referenzzentrum erwogen werden. Auch die Chartis-Messung bietet technische Hürden und z.T. Probleme in der Interpretation der gewonnenen Fluss- und Druckkurven [4, 5, 6]. Mit einer Lernkurve ist zu rechnen. Dennoch ist die Studie ein Meilenstein und bringt Klarheit in einen wichtigen Evaluationsschritt von Emphysempatienten für die endoskopische Therapie.

\footnotetext{
Literatur

1. Klooster K, ten Hacken NH, Hartman JE et al. NEJM 2015;373:2325-35.

2. Davey C, Zoumot Z, Jordan S et al. Lancet 2015;386:1066-73.

Koenigkam-Santos M, Puderbach M et al. Eur J Radiol 2012;81:4161-6.

4. Gesierich W, Samitas K, Behr J. Thorax 2014;69:289-90.

5. Shah PL, Herth FJ. Thorax 2014;69:290-1.

6. Gesierich W, Samitas K, Reichenberger F, Behr J. Eur Respir J 2016;47:1657-67.
}

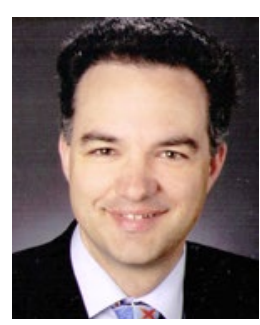

Dr. med. Wolfgang Gesierich

Asklepios-Fachkliniken München-Gauting, Comprehensive Pneumology Center Mitglied im Deutschen Zentrum für Lungenforschung (DZL) Robert-Koch-Allee 2, 82131 Gauting w.gesierich@asklepios.com 